\title{
Association of Socioeconomic Status Measures With Physical Activity and Subsequent Frailty in Older Adults
}

Mark Kheifets ( $\square$ mark.kheifets@outlook.com )

Rabin Medical Center

Abigail Goshen

Tel Aviv University

Uri Goldbourt

Tel Aviv University

Guy Witberg

Rabin Medical Center

Alon Eisen

Rabin Medical Center

Ran Kornowski

Rabin Medical Center

Yariv Gerber

Tel Aviv University

\section{Research Article}

Keywords: exercise, sports, prevention, frailty

Posted Date: November 11th, 2021

DOI: https://doi.org/10.21203/rs.3.rs-1056571/v1

License: (9) (i) This work is licensed under a Creative Commons Attribution 4.0 International License. Read Full License 


\section{Abstract}

Background: Despite increased recognition, frailty remains a significant public health challenge.

Methods: Using a population-based cohort of older adults, this study examined the relationship between socioeconomic status (SES) factors, physical activity and frailty. The study included 1,799 participants (mean [SD], age 75[6]; 53\% women) from the "National Health and Nutrition Survey of Older Adults Aged 65 and Over in Israel", conducted in 2005-2006. A follow-up interview was performed 12-14 years later in a subgroup of 601 subjects (mean [SD], age 84[4]; 56\% women). Extensive data including self-reported leisure-time physical activity (LTPA) and SES measures were assessed at baseline. Frailty was measured at follow-up.

Results: All SES measures were strongly and positively associated with LTPA (all $p<0.001$ ). Eighty-two participants (14\%) were classified as frail at follow-up. After age and sex adjustment and accounting for attrition bias using inverse probability weighting, baseline LTPA (OR=2.77, 95\% Cl: 1.57-4.90, for inactivity; $\mathrm{OR}=1.41,95 \% \mathrm{Cl}: 0.75-2.68$, for insufficient activity, compared with sufficient activity, $\mathrm{P}_{\text {trend }}<0.001$ ) was inversely associated with incident frailty. The association persisted after further adjustment for SES and comorbidity.

Conclusion: Among older individuals, multiple SES measures were positively associated with LTPA, which was a strong predictor of lower subsequent frailty risk.

\section{Introduction}

The term "frailty" is used to describe a range of conditions in older people, including general debility and cognitive impairment [1]. It is in fact a dynamic state, affecting an individual who experiences losses in one or more domains of human functioning (physical, psychological, cognitive and social) caused by the influence of a range of variables and which increases the risk of adverse outcomes [2-3]. The relationships between demographic, socioeconomic status (SES), health-related, nutritional, and lifestyle factors and frailty are well established [4]. There is evidence to support the notion that neighborhood structural characteristics and social processes contribute to the development of frailty [5]. Moreover, there is an inverse association between frailty and both level of education and income [3-4]. Given the increased recognition and awareness of frailty as a significant public health challenge, and its association with adverse health outcomes, prompt recognition, rapid diagnosis and both effective and efficient treatment are warranted [6-7]. Although the critical time window for interventions has not yet been clearly established, as with various chronic diseases, primary prevention is the cornerstone of treatment in frail adult population. Raising awareness about the risk of the disabling cascade, providing the necessary knowledge to actively prevent, and improving access to care to favor optimal aging represent crucial steps to undertake [6]. The role of physical activity in the prevention and progression of frailty syndrome has been extensively researched, and is becoming increasingly well understood [7]. Even though existing evidence base is too inconsistent to recommend the optimal mode of a single physical 
activity or a single dietary regime for the prevention of frailty, there is emerging evidence for the synergistic benefits of combined physical activity and nutritional interventions for the older person living with frailty [7-9]. In regard to physical exercise, it improves the physical (cardiorespiratory function, muscle function, flexibility), cognitive and psychosocial state of frail individuals and consequently reduces the risk of adverse health outcomes, including mortality [10-11]. Although the effects of education, income and physical activity on frailty are well established, their influence on each other have yet to be studied. The aim of this present research was to evaluate the association of education and income, as well as neighborhood SES, on physical activity and subsequent frailty in older adults. Specifically, we utilized an extensive database of nearly 2,000 Israeli citizens aged $\geq 65$ years.

\section{Methods}

\section{Study Design and Setting}

The study is a prospective cohort study investigating the role of sociodemographic, medical, and psychosocial variables in older adults. Details of the study methods have been previously reported [12]. Briefly, during 2005-2006, 1,799 older adults (mean [SD], age 75[6] years) participated in the First National Health and Nutrition Survey of Older Adults Aged 65 and over in Israel ('Mabat Zahav'). The study was led

by the Israel Center for Disease Control and the Nutrition Department of the Israel Ministry of Health. Data were obtained via a personal interview in the interviewee's place of residence (own home or retirement home) using a structured questionnaire. The data collected in the survey (T1) included information regarding health and nutrition status, health behaviors (physical activity, alcohol consumption, medication use and use of nutrition supplements), knowledge and attitudes regarding nutrition and utilization of health services. During 2017-2019, a follow-up interview was conducted among 601 past participants (mean [SD], age 84[4] years). The follow-up questionnaire (T2) duplicates most parts of the original ( $\mathrm{T} 1)$ interview. In addition, measurements pertaining to frailty status were performed according to Fried and colleagues' Frailty Phenotype framework (FP) [13].

\section{Data collection}

\section{SES measures}

Individual SES data were provided at baseline by self-report and included years of education, family status, house-hold income (low < 5,254 NIS; Intermediate 5,255-10,459 NIS; High > 10,460 NIS per month) and current employment status (salaried, unsalaried or volunteer vs. none). Neighborhood SES was estimated according to home address, via an index developed and validated by the Israel Central Bureau of Statistics. This index allows the classification of small geographic units into SES categories, based on socioeconomic measures (i.e., demographics, living standards, education, employment, social welfare benefits) from the 2008 National Census. Neighborhood SES scores were rated on a 20-point scale. A composite SES score (exposure variable) was calculated as follows. Education, income, and neighborhood SES were transformed into standardized z-scores, which were reversely coded such that 
negative values indicating better SES and positive values indicating worse SES. The composite SES score was computed by averaging the summed total.

\section{Clinical variables}

Self-rated health, a single question measure rated on a 4-point scale (4 -very good health). Mini mental state examination (MMSE) was adjusted for age and education.

\section{Primary exposure assessment}

Physical activity: LTPA was self-reported during both baseline and follow-up interviews, based on a standard questionnaire (for full questionnaire see Israeli Ministry of Health website - English version available). In 2 sets of questions, participants were asked about their PA habits. One set referred to vigorous-intensity activity and another set addressed any type of moderate PA that lasted at least 10 minutes. Participants reported the frequency (times per week) and average time they devoted to each specific activity, as follows: walking outdoors or on a treadmill, jogging, swimming, bike riding or stationary cycling, light exercise (such as yoga, the Feldenkrais method, the Alexander technique, light gymnastics), body shaping, and strength training; an "other activity" option was also offered [14]. Based on reported total weekly time of PA and intensity, participants were classified into 3 PA categories according to the official American College of Sports Medicine (ACSM) guideline [15]: sufficiently active, insufficiently active, or inactive. Individuals who performed moderate PA for at least 150 minutes per week or a vigorous-intensity activity for at least 75 minutes per week or a combination of the two were classified as sufficiently active; those who engaged in PA but in a lesser amount than these definitions were classified as insufficiently active; and those who reported no activity or activity less than once a week were classified as inactive.

\section{Primary outcome assessment}

Frailty: Frailty was assessed at T2 by the Fried's Phenotype Model [13]. Using this instrument, frailty was identified by the presence of three or more of the following components: 1. Shrinking. weight loss, unintentional, of more than $4.5 \mathrm{Kg}$, or more than $5 \%$ of body weight, in the previous year; 2 . Weakness: grip strength in the lowest $20 \%$ (adjusted for sex and body mass index); 3. Poor endurance and energy. as indicated by self-report of exhaustion; 4 . Slowness: the slowest $20 \%$ of the participants in the sample, based on time of a 5-meter walk (adjusted for sex and standing height); 5. Low physical activity level: a weighted score of kilocalories expended per week, based on the physical activity scale for the elderly (PASE) questionnaire [16]. The lowest quintile of physical activity was identified for each gender.

\section{Statistical analysis}

Analyses were performed using IBM SPSS V.27 and R version 3.4.4 (R Development Core Team). Descriptive statistics of baseline characteristics of study participants by LPTA categories were compared by chi-squared test for categorical variables and analysis of variance for continuous variables. Logistic regression models were constructed in order to assess the role of the physical activity in long-term incidence of frailty. Logistic regression models were also used to assess the association between SES 
and incidence of frailty, before and after adjustment for LTPA. Missing values for covariates and individual components of the FP were imputed using multiple imputation methodology. Five datasets were created, with missing values replaced by imputed values based on models incorporating demographic, socioeconomic, psychosocial, and clinical variables. The results of these datasets were then combined using Rubin's rules. Of the 1,799 participants in the initial survey, many were unable or unavailable to participate in the second interview. Because frailty could not be assessed among the latter group, selection bias is introduced. This bias was addressed through an adaptation of a marginal structural model, applying inverse probability weights [17]. The weights were calculated using logistic regression model to assess the probability of original participants to participate in T2. Each observation was then weighted by the reciprocal (i.e., the inverse) of the predicted probability of participating at T2.

\section{Results}

Baseline Characteristics at study entry, categorized by physical activity, are shown in Table I. Sufficiently active participants were younger, predominantly male, and mostly married. They had lower body mass index (BMI), smoked less, and suffered from less cardiovascular diseases. They had less comorbidities, less functional and mental limitations, and rated their overall health higher, as compared to both inactive and insufficiently active participants.

\section{Table I. Baseline characteristics, categorized by LPTA, at study entry}




\begin{tabular}{|c|c|c|c|c|}
\hline \multirow[b]{2}{*}{ Variable } & \multicolumn{4}{|c|}{ LPTA categories } \\
\hline & $\begin{array}{l}\text { Inactive } \\
(n=734)\end{array}$ & $\begin{array}{l}\text { Insufficiently } \\
\text { active } \\
(n=506)\end{array}$ & $\begin{array}{l}\text { Sufficiently } \\
\text { active } \\
(n=559)\end{array}$ & $\begin{array}{l}P \\
\text { value }\end{array}$ \\
\hline Age, years, mean (SD) & $75.1(6.6)$ & $74.8(6.0)$ & $73.9(5.8)$ & 0.002 \\
\hline Female, n (\%) & $\begin{array}{l}439 \\
(59.8)\end{array}$ & $284(56.1)$ & $235(42.0)$ & $<0.001$ \\
\hline Employment, n (\%) & $\begin{array}{l}176 \\
(23.9)\end{array}$ & $106(20.9)$ & $113(20.2)$ & 0.097 \\
\hline Living alone $\mathrm{n}(\%)$ & $183(24.9)$ & $122(24.1)$ & $122(21.8)$ & 0.417 \\
\hline Married, n (\%) & $\begin{array}{l}427 \\
(58.5)\end{array}$ & $310(61.6)$ & $403(73.1)$ & $<0.001$ \\
\hline $\begin{array}{l}\text { Self-rated health- good/ very good, } n \\
(\%)\end{array}$ & $\begin{array}{l}296 \\
(40.3)\end{array}$ & $281(55.5)$ & $409(73.2)$ & $<0.001$ \\
\hline Cardiovascular disease, $\mathrm{n}(\%)$ & $\begin{array}{l}296 \\
(40.3)\end{array}$ & $171(33.8)$ & $180(32.2)$ & 0.002 \\
\hline Hypertension, n (\%) & $\begin{array}{l}288 \\
(40.1)\end{array}$ & $209(41.6)$ & $254(45.7)$ & 0.127 \\
\hline Comorbidities, n (\%) & & & & $<0.001$ \\
\hline 0 & $75(10.2)$ & $68(13.4)$ & $82(14.7)$ & \\
\hline $1-3$ & $\begin{array}{l}538 \\
(73.3)\end{array}$ & $366(72.3)$ & $435(77.8)$ & \\
\hline$\geq 4$ & $\begin{array}{l}121 \\
(16.5)\end{array}$ & $72(14.2)$ & $42(7.5)$ & \\
\hline BMI $\left(\mathrm{kg} / \mathrm{m}^{2}\right)$, mean (SD) & $30.3(5.4)$ & $29.1(4.5)$ & $28.0(4.0)$ & $<0.001$ \\
\hline Adjusted MMSE score, mean (SD) & $30.5(3.6)$ & $30.8(3.8)$ & $30.8(3.8)$ & 0.213 \\
\hline Functional limitations, n (\%) & & & & $<0.001$ \\
\hline No functional limitations & $\begin{array}{l}470 \\
(64.0)\end{array}$ & $426(84.2)$ & $514(91.9)$ & \\
\hline Some functional limitations & $\begin{array}{l}215 \\
(29.3)\end{array}$ & $66(13.0)$ & $43(7.7)$ & \\
\hline Severe functional limitations & $49(6.7)$ & $14(2.8)$ & $2(0.4)$ & \\
\hline GHQ score, mean (SD) & $6.8(5.5)$ & $6.7(2.8)$ & $5.6(3.1)$ & $<0.001$ \\
\hline Smoking status, n (\%) & & & & 0.033 \\
\hline Smoking Today & $97(13.2)$ & $50(9.9)$ & $49(8.8)$ & \\
\hline
\end{tabular}




\begin{tabular}{llll} 
Former Smoker & 240 & $168(33.2)$ & $213(38.1)$ \\
& $(32.7)$ & & \\
\hline Never Smoker & 397 & $288(56.9)$ & $297(53.1)$ \\
& $(54.1)$ & & \\
\hline
\end{tabular}

Values are expressed as $\mathrm{n}(\%)$ or mean \pm SD.

Abbreviations: LPTA, leisure time physical activity; BMI, body mass index; MMSE, mini-mental state examination; $\mathrm{GHQ}$, general health questionnaire.

Table II. Baseline characteristics, across LPTA categories, among T2 participants

\begin{tabular}{|c|c|c|c|c|}
\hline \multicolumn{5}{|c|}{ Baseline LPTA categories } \\
\hline Baseline variable & $\begin{array}{l}\text { Inactive } \\
(n=191)\end{array}$ & $\begin{array}{l}\text { Insufficiently } \\
\text { active } \\
(n=178)\end{array}$ & $\begin{array}{l}\text { Sufficiently active } \\
(n=232)\end{array}$ & $P$ value \\
\hline Age, years, mean (SD) & $72.0(4.8)$ & $72.4(4.7)$ & $71.7(4.5)$ & 0.301 \\
\hline Female, n (\%) & $\begin{array}{l}127 \\
(66.5)\end{array}$ & $109(61.2)$ & $100(43.1)$ & $<0.001$ \\
\hline Living alone $\mathrm{n}(\%)$ & $40(20.9)$ & $41(23.0)$ & $31(13.4)$ & 0.027 \\
\hline Married, n (\%) & $\begin{array}{l}126 \\
(66.0)\end{array}$ & $127(71.3)$ & $190(81.9)$ & $<0.001$ \\
\hline Comorbidities, n (\%) & & & & 0.346 \\
\hline 0 & $21(11.0)$ & 19 (10.7) & $31(13.4)$ & \\
\hline $1-3$ & $\begin{array}{l}150 \\
(94.2)\end{array}$ & $147(82.6)$ & $188(81.0)$ & \\
\hline$\geq 4$ & $20(10.5)$ & $12(6.7)$ & $13(5.6)$ & \\
\hline $\mathrm{BMI}\left(\mathrm{kg} / \mathrm{m}^{2}\right)$, mean (SD) & $30.1(4.8)$ & $29.0(4.0)$ & $28.1(3.9)$ & $<0.001$ \\
\hline $\begin{array}{l}\text { Adjusted MMSE score, mean } \\
\text { (SD) }\end{array}$ & $31.0(4.3)$ & $30.8(2.8)$ & $31.2(2.8)$ & 0.543 \\
\hline No functional limitations, $\mathrm{n}(\%)$ & 164(85.9) & 173(97.2) & 229(98.7) & $<0.001$ \\
\hline
\end{tabular}

Values are expressed as $n(\%)$ or mean $\pm S D$.

Abbreviations: LPTA, leisure time physical activity; BMI, body mass index; MMSE, mini-mental state examination. 
Baseline SES measures, in the entire cohort and in a subgroup of T2 participants, are shown in table III. Sufficiently active participants were more educated, had a higher household income, and lived in neighborhoods with a higher SES score, as compared to both inactive and insufficiently active participants.

Table III. Baseline SES measures, categorized by baseline LTPA, in the total sample and among T2 participants only

\begin{tabular}{|c|c|c|c|c|}
\hline \multicolumn{5}{|c|}{ Baseline LTPA Level among T1 participants $(n=1799)$} \\
\hline Baseline SES measure & $\begin{array}{l}\text { Inactive } \\
(n=734\end{array}$ & $\begin{array}{l}\text { Insufficiently } \\
\text { active } \\
(n=506)\end{array}$ & $\begin{array}{l}\text { Sufficiently } \\
\text { active } \\
(n=559)\end{array}$ & $\begin{array}{l}\mathrm{P} \\
\text { value }\end{array}$ \\
\hline Education, years, mean (SD) & $8.7(5.5)$ & $11.1(4.7)$ & $12.5(4.5)$ & $<0.001$ \\
\hline Household income category, n (\%) & & & & $<0.001$ \\
\hline Low & $\begin{array}{l}381 \\
(51.9)\end{array}$ & $201(39.7)$ & $189(33.8)$ & \\
\hline Intermediate & $\begin{array}{l}126 \\
(17.1)\end{array}$ & $121(23.9)$ & $178(31.8)$ & \\
\hline High & 223(30.3) & $182(35.9)$ & $190(33.9)$ & \\
\hline $\begin{array}{l}\text { Neighborhood SES score, mean } \\
\text { (SD) }\end{array}$ & $9.4(4.2)$ & $11.1(4.2)$ & $11.5(3.9)$ & $<0.001$ \\
\hline \multicolumn{5}{|c|}{ Baseline LTPA Level among T2 participants $(n=601)$} \\
\hline Baseline SES measure & $\begin{array}{c}\text { Inactive } \\
(\mathrm{n}=191)\end{array}$ & $\begin{array}{l}\text { Insufficiently } \\
\text { active } \\
(n=178)\end{array}$ & $\begin{array}{l}\text { Sufficiently } \\
\text { active } \\
(n=232)\end{array}$ & $\begin{array}{l}\mathrm{P} \\
\text { value }\end{array}$ \\
\hline Education, years, mean (SD) & $8.4(5.6)$ & $12.0(4.7)$ & $12.4(4.3)$ & $<0.001$ \\
\hline Household income category, n (\%) & & & & $<0.001$ \\
\hline Low & $94(49.2)$ & $61(34.3)$ & $56(24.1)$ & \\
\hline Intermediate & $31(16.2)$ & $45(25.3)$ & $81(34.9)$ & \\
\hline High & $66(34.6)$ & $72(40.4)$ & $95(40.9)$ & \\
\hline $\begin{array}{l}\text { Neighborhood SES score, mean } \\
\text { (SD) }\end{array}$ & $9.4(4.4)$ & $11.5(4.5)$ & $12.0(4.1)$ & $<0.001$ \\
\hline
\end{tabular}


Values are expressed as $\mathrm{n}(\%)$ or mean \pm SD.

Abbreviations: LTPA, leisure time physical activity; SES, socioeconomic status.

Household income categories: low < 5,254 NIS. Intermediate 5,255-10,459 NIS. High > 10,460 NIS.

Eighty-two participants (14\%) were classified as frail at follow-up. Frailty components among frail participants are shown in figure $\mathrm{I}$.

Odds ratios (OR) of frailty development, according to baseline LPTA categories, are shown in table IV. After adjustment for age and sex, decreasing LTPA levels were strongly associated with frailty incidence. Further adjustment for a composite SES score and comorbidity burden attenuated, but did not eliminate, the association. The distribution [mean z-score (SD); inversely coded] of the composite SES score by baseline LTPA categories was as follows: -.32 (-.31) for sufficiently active, -.19 (0.57) for insufficiently active, and .34(0.69) for inactive $(P<.001)$. Adjusted for age and sex, the OR (95\% confidence interval) for frailty at follow up associated with lower SES was 2.25 (1.62-3.12). Further adjustment for PA attenuated the OR to 1.87 (1.01-3.46). Thus, approximately $20 \%$ of the SES-frailty association is attributable to PA.

Table IV. Odds ratios of frailty development, categorized by baseline LPTA, among study participants

\begin{tabular}{|lllll|}
\hline Adjustment & \multicolumn{2}{l}{ Physical activity categories } & P for trend \\
\hline Frailty phenotype & $\begin{array}{l}\text { Sufficiently active } \\
(\mathrm{n}=232)\end{array}$ & $\begin{array}{l}\text { Insufficiently active } \\
(\mathrm{n}=178)\end{array}$ & $\begin{array}{l}\text { Inactive } \\
(\mathrm{n}=191)\end{array}$ & \\
& & & & \\
\hline Model 1 & 1 & $1.41(0.75-2.68)$ & $2.77(1.57-4.90)$ & $<0.001$ \\
\hline Model 2 & 1 & $1.32(0.70-2.53)$ & $1.87(1.01-3.46)$ & 0.04 \\
\hline Model 3 & 1 & $1.26(0.65-2.45)$ & $1.71(0.90-2.24)$ & 0.06 \\
\hline
\end{tabular}

Abbreviations: LPTA, leisure time physical activity; SES, socioeconomic status.

Model 1: age and sex. Model 2: further adjusted for SES composite score. Model 3: further adjusted for comorbidities.

Comorbidities: heart attack, cardiac insufficiency, other heart disease, stroke, cataract, glaucoma, chronic renal failure, cancer, Alzheimer's disease, Parkinson's disease, asthma, other lung disease, diabetes, osteoporosis, dyslipidemia, hypertension.

\section{Discussion}

Population aging is poised to become one of the most significant social transformations of the twentyfirst century, with implications for nearly all sectors of society [18]. While one 70-year-old person may 
enjoy good health that enables them to remain active and to live without much health care support or intervention, a peer of the same age may face multiple chronic morbidities that cause significant disability and require frequent medical interventions or various support resources. Level of income, educational attainment and physical activity may help to distinguish between the two. Approximately a quarter of individuals aged $>85$ years are living with frailty and as such the identification of those who are frail is a public health priority ${ }^{7}$.

In this large prospective registry of 1,799 older adults, 601 of which were interviewed for the second time after nearly 15 years, sufficient LTFA was associated with lower rates of frailty, as compared to both relative and absolute inactivity. Furthermore, education and income, as well as area-based SES, served as strong predictive factors for physical activity frequency, and subsequent development of frailty. Similar to findings described by Gerber et al [19], these multiple and multidimensional SES measures emphasize the powerful association between neighborhood SES and LTPA.

Our findings are consistent with earlier studies [2-4], which showed that physical activity is a key factor in both prevention and deceleration of the inevitable progression of an already established state of frailty. As was described by Van Oostrom et al [3], being physically active decreases the risk of being frail on all four domains - physical, psychological, cognitive, and social. Furthermore, as was described by Woolford et al [7], the degree to which one is physically active can directly contribute to the frailty syndrome in several ways. First, physical inactivity can lead to a myriad of diverse chronic health issues, including cardiovascular disease, cerebrovascular disease, type two diabetes, depression and dementia. Second, loss of muscle strength and progression to sarcopenia, may lead to imbalance, poor posture and eventual state of recurrent falls, with its potential adverse sequelae in the form of bone fracture, hospital admission and further decompensation. Combination of aerobic, resistance, balance, flexibility, and functional based exercise can help prevent the above. Therefore, it is crucial to follow a strict exercise prescription in order to avoid, and in some cases even reverse, frailty. As described by bray et al [20], optimal frequency for multi-component training is $2-3$ times per week. In addition, it is critical that individuals engage in exercise at an intensity that will elicit a fitness benefit by overloading the desired physiological system, causing it to adapt to meet the needs of the exercise demand. Duration of each exercise may vary, with optimal time of 30-60 minutes per session.

Concomitantly to physical activity, demographic and socioeconomic factors such as education and household income serve as overwhelmingly important risk factors for the prediction of frailty [3-4]. As such, they should be taken under great consideration by educators, medical providers, and both local and government officials. Awareness for health and physical activity should be taught from the earliest age possible, and accessibility to fitness facilities in low socioeconomic neighborhoods is of paramount importance. Nutrition is yet another key factor in the development of frailty, and its quality is directly connected to SES and income. As was described by French et al [21], lower income households purchase less healthful foods compared with higher income households. Food purchasing patterns may mediate income differences in dietary intake quality. Malnutrition is highly prevalent among older adults and 
associated with a general decline in physical and mental functioning, higher hospitalization rate and increased mortality [22]. As emphasized in several clinical guidelines [23], adequate caloric intake, and both protein and vitamin $D$ supplementation, when indicated, are essential for the prevention and progression of frailty in older adults. As was so eloquently described by WOO et al [24], neither nutrition nor frailty are topics that the majority of physicians and researchers are familiar with, but considering the continued increase in life expectancy on the global level, much needs to be done to raise awareness of the clinical importance of both. This approach represents true patient centered care in directing the goal of health promotion and clinical care towards maintenance of physical and cognitive function.

\section{Limitations}

Although all data were collected prospectively, the SES-LTPA association was processed using a cross sectional analysis. Second, we did not have information regarding the participants physical activity during follow-up, nor did we assess frailty at baseline. We assumed that there were a very small number of frail participants at baseline, who attended the follow-up visit more than a decade later. Third, our data relied on self-reported questionnaires, without physical examination, laboratory workup or further imaging studies. Finally, only $\sim 1 / 3$ of the original participants were re-interviewed, leading to a relatively small sample size at follow-up, and ultimately resulting in attrition bias. Nevertheless, this current study presented a well-defined cohort of nationally representative older adults with repeated measurements of aging indicators, evaluation of frailty using the Fried criteria (rather than using a frailty index), rich dataset with multiple and multi-level SES and clinical measures and a detailed LTPA questionnaire that was validated against different outcomes in previous studies, and an up-to-date statistical analysis to minimize the effect of attrition and missing data.

\section{Conclusions}

Constant increase in life expectancy, primarily due to advancements in medicine and technology, puts frailty on the list of most burning medical issues of the $21^{\text {st }}$ century. Physical activity is an effective and generally inexpensive form of both prevention and treatment of frailty. Hence, encouragement and active promotion of LTFA should be a top priority for all medical practitioners, with an emphasis on those working with underprivileged populations.

\section{Abbreviations}

The following abbreviations are used in this manuscript:

SES Socioeconomic status

LTPA Leisure-time physical activity

FP Frailty Phenotype framework

MMSE Mini mental state examination 
ACSM American College of Sports Medicine

BMI Body mass index

PASE Physical activity scale for the elderly

GHQ General health questionnaire

OR Odds ratio

\section{Declarations}

Ethics approval and consent to participate - Informed consent was obtained at each examination phase and the study was approved by the Helsinki Committee of Chaim Sheba Medical Center at Tel Hashomer and the ethics committee at Tel-Aviv University. The consent obtained from the study participants was written. The study protocol was in accordance to guidelines of the Institutional Review Boards.

Consent for publication - Not applicable.

Availability of data and materials - The datasets generated and analyzed during the current study are available in the "Mabat Zahav" repository (www.health.gov.il)

Competing interests - None

Funding - None

\section{Authors' Contributions:}

1. Mark Kheifets wrote the paper.

2. Yariv Gerber conceived and designed the analysis and reviewed the paper

3. Abigail Goshen collected data and preformed the analysis.

4. Uri Goldbourt collected data and contributed to the analysis.

5. Guy Wittberg collected data and reviewed the paper.

6. Alon Eisen contributed to the analysis and reviewed the paper

7. Ran Kornowski contributed to the analysis and reviewed the paper

\section{References}

1. Uchmanowicz I, Jankowska-Polańska B, Wleklik M, et al. Frailty Syndrome: Nursing Interventions. SAGE Open Nurs. 2018;4:2377960818759449. Published 2018 Mar 15.

doi:10.1177/2377960818759449 
2. Gobbens RJ, Luijkx KG, Wijnen-Sponselee MT, et al. Toward a conceptual definition of frail community dwelling older people. Nurs Outlook. 2010;58(2):76-86.

doi:10.1016/j.outlook.2009.09.005

3. van Oostrom SH, van der A DL, Rietman $\mathrm{ML}$, et al. A four-domain approach of frailty explored in the Doetinchem Cohort Study. BMC Geriatr. 2017;17(1):196

4. Mello Ade C, Engstrom EM, Alves LC. Health-related and socio-demographic factors associated with frailty in the elderly: a systematic literature review. Cad Saude Publica. 2014;30(6):1143-1168. doi:10.1590/0102-311x00148213

5. Caldwell JT, Lee H, Cagney KA. Disablement in Context: Neighborhood Characteristics and Their Association With Frailty Onset Among Older Adults. J Gerontol B Psychol Sci Soc Sci. 2019;74(7):e40-e49. doi:10.1093/geronb/gbx123

6. Cesari M, Prince M, Thiyagarajan JA, et al. Frailty: An Emerging Public Health Priority. J Am Med Dir Assoc. 2016;17(3):188-192. doi:10.1016/j.jamda.2015.12.016

7. Woolford SJ, Sohan O, Dennison EM, et al. Approaches to the diagnosis and prevention of frailty. Aging Clin Exp Res. 2020;32(9):1629-1637. doi:10.1007/s40520-020-01559-3

8. Bloom I, Shand C, Cooper C et al. Diet Quality and Sarcopenia in Older Adults: A Systematic Review. Nutrients. 2018;10(3):308. Published 2018 Mar 5. doi:10.3390/nu10030308

9. Apóstolo J, Cooke R, Bobrowicz-Campos E, et al. Effectiveness of interventions to prevent pre-frailty and frailty progression in older adults: a systematic review [published correction appears in JBI Database System Rev Implement Rep. 2018 May;16(5):1282-1283]. JBI Database System Rev Implement Rep. 2018;16(1):140-232. doi:10.11124/JBISRIR-2017-003382

10. Theou O, Stathokostas L, Roland KP, et al. The effectiveness of exercise interventions for the management of frailty: a systematic review. J Aging Res. 2011;2011:569194. Published 2011 Apr 4. doi:10.4061/2011/569194

11. Rossi PG, Carnavale BF, Farche ACS, et al. Effects of physical exercise on the cognition of older adults with frailty syndrome: A systematic review and meta-analysis of randomized trials. Arch Gerontol Geriatr. 2021;93:104322. doi:10.1016/j.archger.2020.104322

12. Goshen A, Goldbourt $U$, Shohat $T$, et al. Diet quality in relation to healthy ageing: the Israeli Longitudinal Study on Aging (ILSA)-a study protocol. BMJ Open. 2019;9(4):e024673. Published 2019 Apr 20. doi:10.1136/bmjopen-2018-024673

13. Fried LP, Tangen CM, Walston J, et al. Frailty in older adults: evidence for a phenotype. J Gerontol $A$ Biol Sci Med Sci. 2001;56(3):M146-M156. doi:10.1093/gerona/56.3.m146

14. Cohen G, Steinberg DM, Keinan-Boker L, et al. Leisure-Time Physical Activity and Cancer Risk Among Older Adults: A Cohort Study. Mayo Clin Proc Innov Qual Outcomes. 2020;4(2):115-125. Published 2020 Apr 6. doi:10.1016/j.mayocpiqo.2019.11.005

15. Thompson PD, Arena R, Riebe D, et al. American College of Sports Medicine. ACSM's new preparticipation health screening recommendations from ACSM's guidelines for exercise testing and 
prescription, ninth edition. Curr Sports Med Rep. 2013;12(4):215-217.

doi:10.1249/JSR.0b013e31829a68cf

16. Washburn RA, Smith KW, Jette AM, et al. The Physical Activity Scale for the Elderly (PASE): development and evaluation. J Clin Epidemiol. 1993;46(2):153-162. doi:10.1016/08954356(93)90053-4

17. Robins JM, Hernán MA, Brumback B. Marginal structural models and causal inference in epidemiology. Epidemiology. 2000;11(5):550-560. doi:10.1097/00001648-200009000-00011

18. Kanasi E, Ayilavarapu S, Jones J. The aging population: demographics and the biology of aging. Periodontol 2000. 2016;72(1):13-18. doi:10.1111/prd.12126

19. Gerber Y, Myers V, Goldbourt U, et al. Israel Study Group on First Acute Myocardial Infarction. Neighborhood socioeconomic status and leisure-time physical activity after myocardial infarction: A longitudinal study. Am J Prev Med. 2011;41(3):266-273. doi:10.1016/j.amepre.2011.05.016

20. Bray NW, Smart RR, Jakobi JM, et al. Exercise prescription to reverse frailty. Appl Physiol Nutr Metab. 2016;41(10):1112-1116. doi:10.1139/apnm-2016-0226

21. French SA, Tangney CC, Crane MM, et al. Nutrition quality of food purchases varies by household income: the SHoPPER study. BMC Public Health. 2019;19(1):231. Published 2019 Feb 26. doi:10.1186/s12889-019-6546-2

22. Ahmed T, Haboubi N. Assessment and management of nutrition in older people and its importance to health. Clin Interv Aging. 2010;5:207-216. Published 2010 Aug 9. doi:10.2147/cia.s9664

23. Mehta P, Lemon G, Hight L, et al. A Systematic Review of Clinical Practice Guidelines for Identification and Management of Frailty. J Nutr Health Aging. 2021;25(3):382-391. doi:10.1007/s12603-020-1549-3

24. Woo J. Nutrition and Frailty. J Nutr Health Aging. 2018;22(9):1025-1027. doi:10.1007/s12603-018$1120-7$

\section{Figures}


70

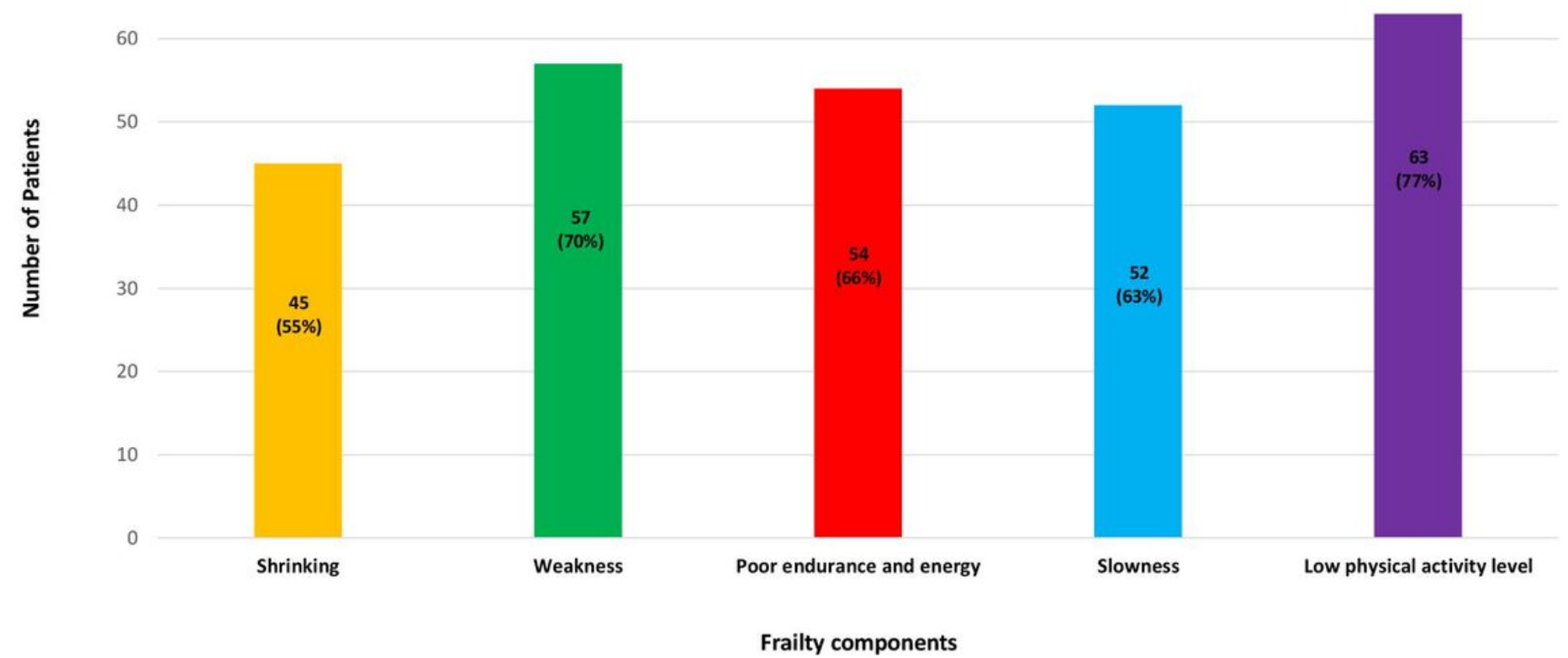

Figure 1

Frailty components among frail participants 